$\begin{array}{cc}\text { ACADEMIA ROMÂNĂ } & \begin{array}{c}\text { Rev. Roum. Chim., } \\ \mathbf{2 0 2 0}, \text { 65(6), 611-616 }\end{array} \\ \text { Revue Roumaine de Chimie } & \text { DOI: 10.33224/rrch.2020.65.6.12 } \\ \text { http://web.icf.ro/rrch/ } & \end{array}$

\title{
POLY(1-VINYLIMIDAZOLE) GRAFTED ON MAGNETIC NANOPARTICLES - ATTAINMENT OF NOVEL NANOSTRUCTURES
}

\author{
Alexandrina $\mathrm{NAN}^{*}$ and Rodica TURCU* \\ National Institute of R\&D for Isotopic and Molecular Technologies, Donat Str. 67-103, 400293 Cluj-Napoca, Roumania
}

New magnetic core shell nanoparticles were synthesized consisting of a magnetite core and a poly(1-vinylimidazole) shell. Magnetite nanoparticles were first coated by bis(methacryloyloxyethoxy)phosphate as a reactive alkene-containing anchor followed by radical polymerization of 1-vinylimidazole. The poly(1vinylimidazole) coated magnetic nanoparticles (MNP-PVIm) represent versatile magnetic nanoplatforms capable of complexing different types of metals. Their chemical structure was investigated by Fourier transform infrared spectroscopy (FTIR) and X-ray photoelectron spectroscopy (XPS). The novel nanostructures MNP-PVIm were used as a promising platform for complexing rare earth

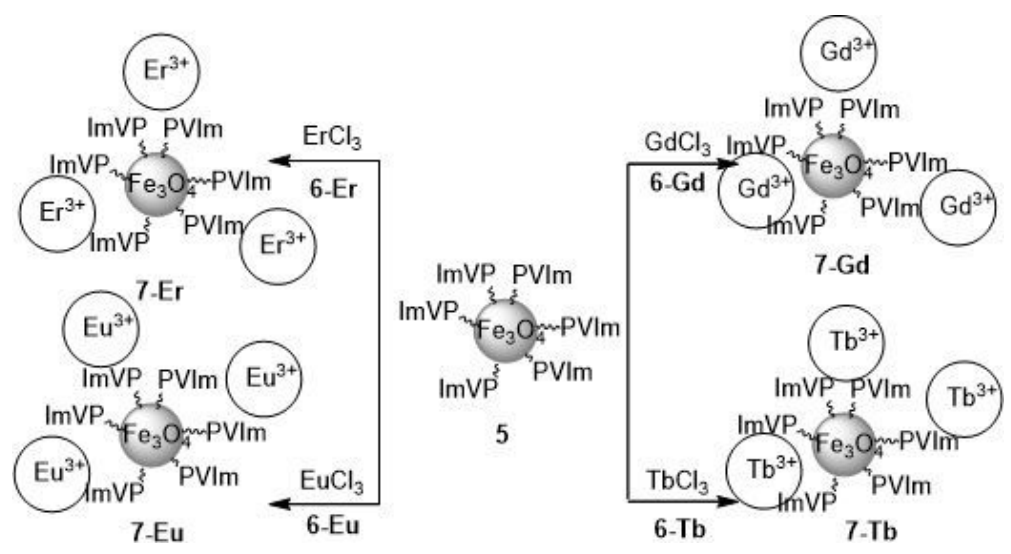
metals, as gadolinium, terbium, erbium and europium. The morphology and the content of rare earth metals were determined by Transmission Electron Microscopy Energy-Dispersive X-ray spectroscopy (TEM-EDX). The magnetization curves of MNP-PVIm demonstrate the superparamagnetic nature of these nanostructures.

\section{INTRODUCTION}

Magnetic nanoparticles (MNPs) based on functionalized polymers have found wide application in medicine as drug delivery systems, magnetic resonance imaging (MRI) or hyperthermia mediators, in separation of biomaterials or noxious compounds and as supports for catalysts. ${ }^{1-7}$

In most of these cases the MNPs have to be covered by anchoring compounds or polymers leading to core-shell nanoparticles. Surface modification by graft polymerization is a versatile method for the molecular design of solid surfaces to enhance their physical and chemical properties for specific applications. It can be accomplished by physical/ chemical adsorption of organic compounds by four major methods: organic vapor condensation, polymer coating, surfactant adsorption and direct silanation ${ }^{8}$. It is advantageous to use starting MNPs that are covered with active stabilizers, which are able to join the ongoing polymerization in a type of copolymerization thus establishing a covalent bond between the polymer and the surface anchors. ${ }^{9-11}$

\footnotetext{
*Corresponding authors: alexandrina.nan@itim-cj.ro or rodica.turcu@itim-cj.ro
} 
Imidazole is a nitrogen-substituted aromatic heterocycle compound with strong affinity for different metal ions. Furthermore, the adsorption capacity would be considerably enhanced via polymerization of imidazole-based monomers. In the literature are many papers reporting about the covering of magnetite with PVIm, but they are using as a polymerization initiator a silica derivative able to initiate the polymerization of VIm at the magnetite surface. ${ }^{12-15}$

The main purpose of this study is the synthesis of new MNPs covered with poly(1-vinylimidazole) (PVIm) shells starting with MNPs coated with bis[2-(methacryloyloxy)ethyl] phosphate as stabilizer capable of joining the polymerization of 1-vinylimidazole (VIm) by means of their $\mathrm{C}=\mathrm{C}$ double bonds. Poly(1-vinylimidazole) is chosen to graft on nanosized magnetic particles, as the resultant MNP-PVIm are anticipated to expand their application. In the case of using silica derivatives capable of initiating the polymerization reaction of VIm on the surface of magnetic nanoparticles, three reaction steps are involved, whereas in our case, the preparation of the nanostructures MNP-PVIm involves two step procedure. That means that the reported procedure is a simplified method in the synthesis of magnetite covered with PVIm. In this respect they are used as excellent platforms for various rare earth (REs) metals, such as gadolinium $(\mathrm{Gd})$, terbium $(\mathrm{Tb})$, erbium (Er) and europium (Eu) complexation. The purpose for the preparation of these core-shell magnetic nanoparticles are their attractive properties including high photostability, absence of blinking, extremely narrow emission lines, large
Stokes shifts, long lifetimes that can be exploited for retarded detection schemes, and facile functionalization strategies. These rare metal containing composites can be moreover exploited for oxidant detection or for implementing potent contrast agents for MRI, which will provide more opportunities for both diagnosis and therapy.

\section{RESULTS}

\section{Synthesis and rare earth metals ion complexation of PVIm coated MNPs as magnetic platforms}

The starting MNP 1 were obtained in a straight forward way by the well-known co-precipitation method from $\mathrm{Fe}$ (II) and $\mathrm{Fe}(\mathrm{III})$ and further stabilization with bis(methacryloyloxyethoxy) phosphate 2. The stabilizer shells of the resulting MNPs 3 already proved to be useful in joining an ongoing polymerisation of functionalized acrylates. ${ }^{16}$ In the present study, they were covered with PVIm by mixing with a solution of VIm 4 in THF in the presence of 2,2'-azobis(2-methylpropionitrile) (AIBN) as initiator (Scheme 1). The resulting MNP-PVIm 5 were easily separated by an external magnet and washed successively with water, ethanol and acetone.

The presence of imidazole ring renders the MNPs 5 as an attractive magnetic nano-platform for complexation of rare earth metals. The complexation took place in water at room temperature in 24 hours, chloride salts were used as precursors for REs ions (Scheme 2).

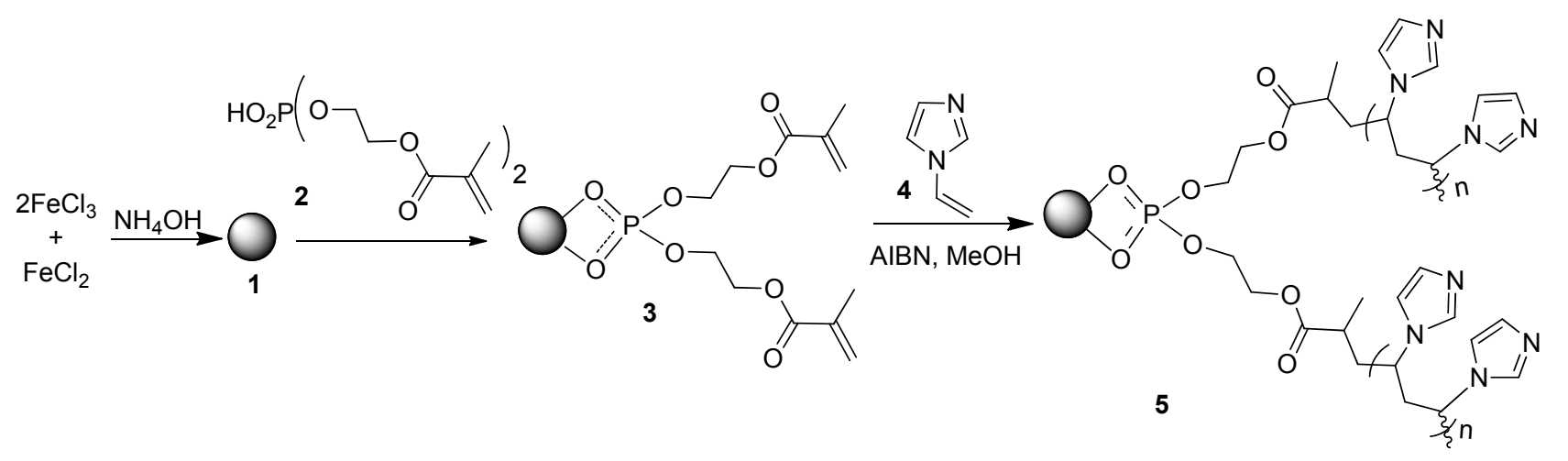

Scheme 1 - Synthesis of PVIm-coated MNPs 5 by primary coverage of MNP $\mathbf{1}$ with bis(methacryloyloxyethoxy) phosphate 2 and subsequent polymerization with VIm 4. 


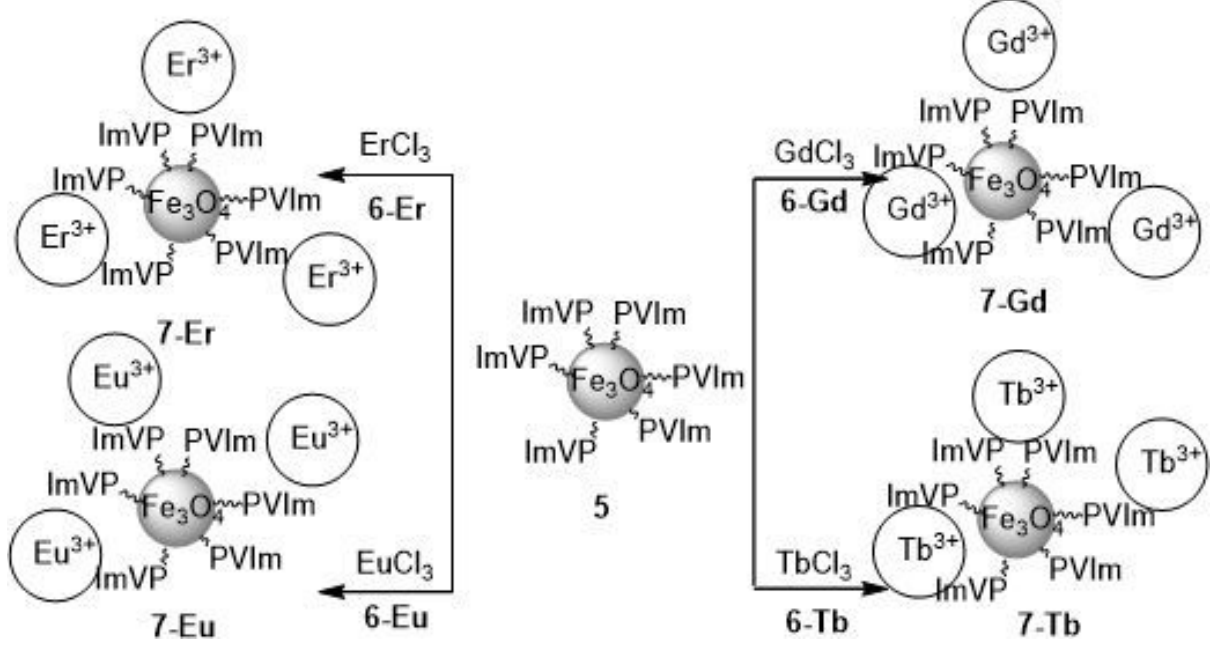

Scheme 2 - Synthesis of REs-chelate PVIm coated magnetic nanoparticles.
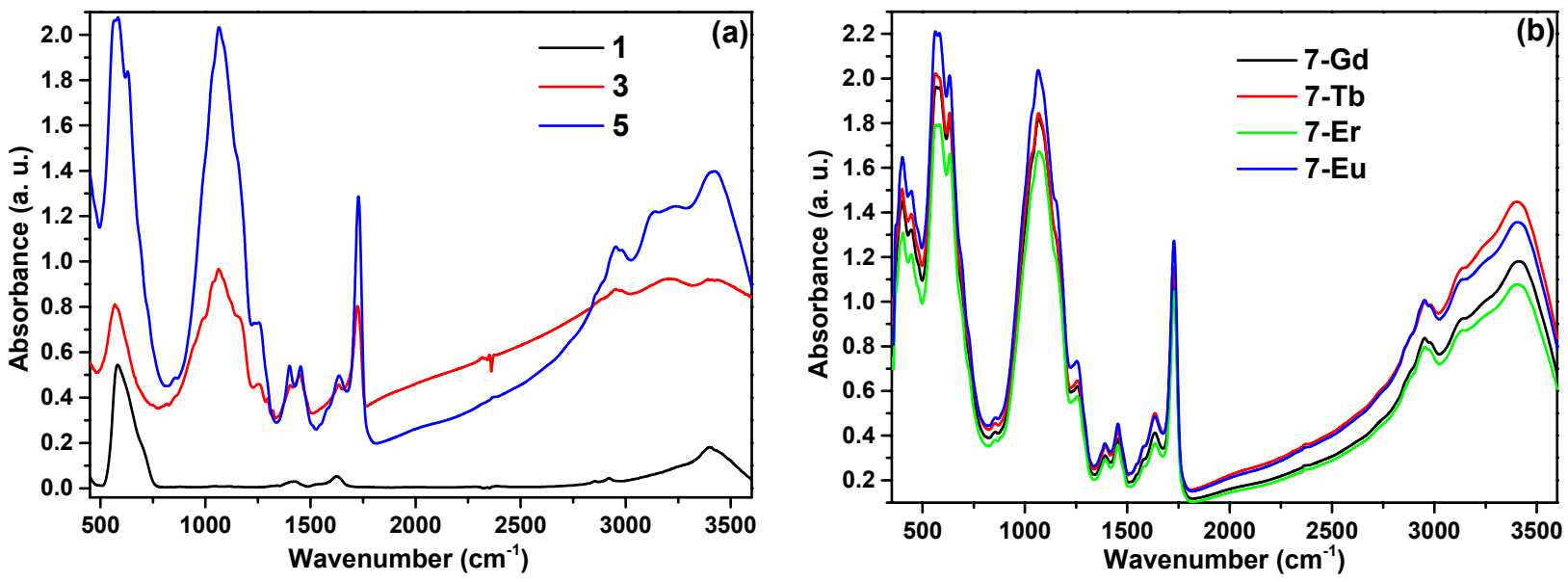

Fig. 1 - FTIR spectra of MNPs 1, MNP 3 and MNPs 5 (a) and FTIR spectra of the MNPs 7-Gd, 7-Tb, 7-Er and 7-Eu (b).

The success of the coating of MNP 1 with bis(methacryloyloxyethoxy) phosphate $\mathbf{2}$ and the synthesis of core-shell nanoparticles MNP 5 was confirmed by FTIR spectroscopy. Thus, the strong absorption band located at around $580 \mathrm{~cm}^{-1}$ in the FTIR spectra of MNPs $\mathbf{1}, \mathbf{3}$ and $\mathbf{5}$ is attributed to the Fe-O bond of magnetite found in the cores (Fig. 1a). Another important band found in both types of MNPs $\mathbf{3}$ and $\mathbf{5}$ is situated at around 1068 $\mathrm{cm}^{-1}$ and is specific for $\mathrm{P}-\mathrm{O}$ bonds stemming from bis(methacryloyloxyethoxy) phosphate 2 . In the case of MNPs $\mathbf{5}$ the adsorption band is more intensive because of the contribution of the $\mathrm{C}-\mathrm{N}$ and $\mathrm{C}=\mathrm{C}$ bond present in the imidazole ring. The peaks at about $1633 \mathrm{~cm}^{-1}$ and $1577 \mathrm{~cm}^{-1}$ corresponding to the $\mathrm{C}-\mathrm{N}$ and $\mathrm{C}-\mathrm{C}$ stretching vibrations on the imidazole ring are observed in IR images of MNPs 5 and MNPs 7-Gd, 7-Tb, 7-Er and 7-Eu. As expected, the band owing to stretching $\mathrm{C}=\mathrm{O}$ vibrations at $1720 \mathrm{~cm}^{-1}$ can be seen in both FTIR spectrum of MNPs 3 and $\mathbf{5}$. The spectrum of magnetic nanoparticles 5 in the range of 2800-3110 $\mathrm{cm}^{-1}$ shows the presence of hydrocarbon groups $-\mathrm{CH}_{2}$ - present in the PVIm chain and to the $=\mathrm{CH}$ - characteristic of VIm ring. ${ }^{13,14,17,18}$ FTIR spectra of the MNPs 7 decorated with different REs metals ions do not show significant differences. In addition, the FTIR spectra of MNPs 7-Gd, 7-Tb, 7-Er and 7-Eu hardly differ from the FTIR spectrum of the precursor MNPs 5.

The PVIm-coated MNPs 5 and their derivatives 7 form suspensions in mixture of methanol and water with partial aggregation. TEM image of MNPs 7 decorated with rare earth metals (Fig. 2) reveals aggregated MNP coated with a relatively high amount of polymer material. Single-particle energy dispersive X-ray (EDX) spectra were analysed for the investigated samples. The results confirm that the MNPs 7 contain REs element, in the following percentage: 7-Gd $-2.9 \% \mathrm{Gd}, \mathbf{7 - T b}$ $3.03 \% \mathrm{~Tb}, 7-\mathbf{E r}-4.11 \% \mathrm{Er}$ and 7-Eu - 3.2 \% Eu. 

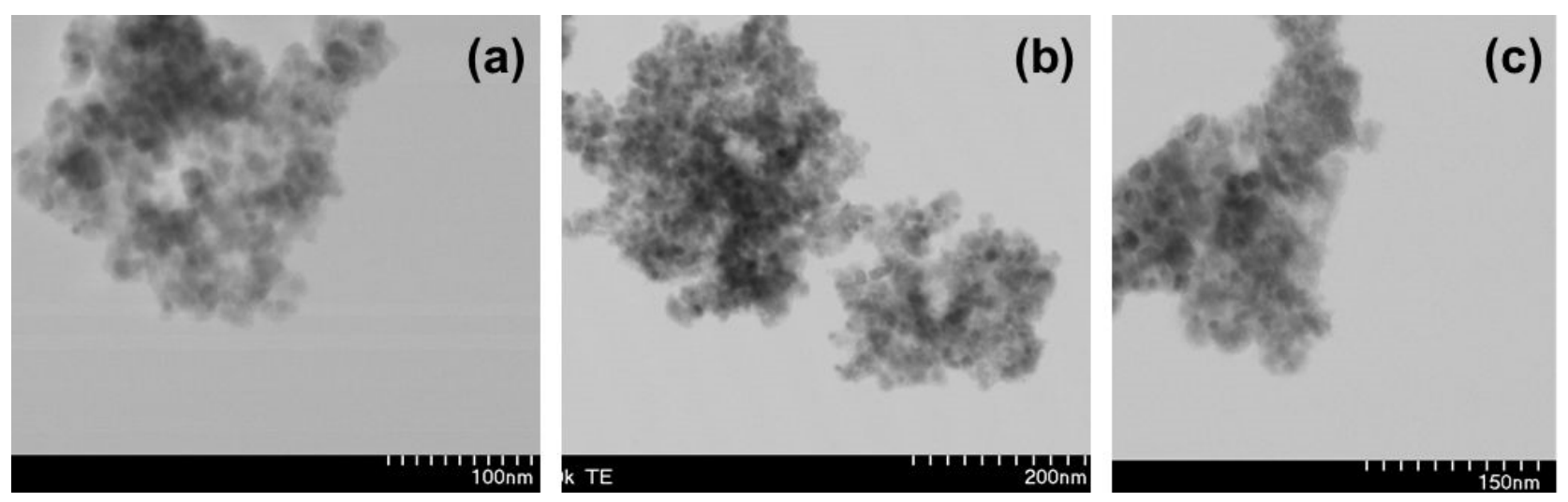

Fig. 2 - TEM image of MNP 7-Tb (a), MNP 7-Er (b) and MNP 7-Eu (c).

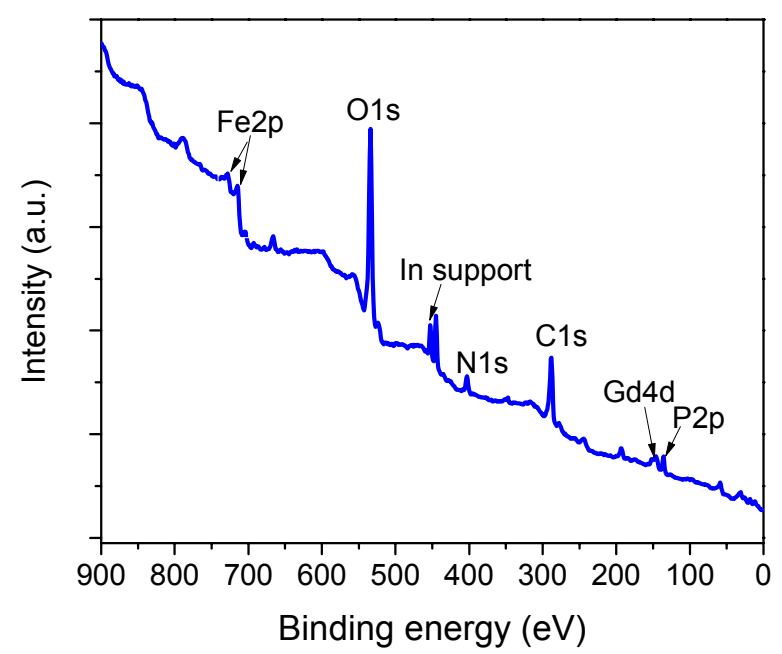

Fig. 3 - XPS survey spectrum of the MNP 7-Gd.
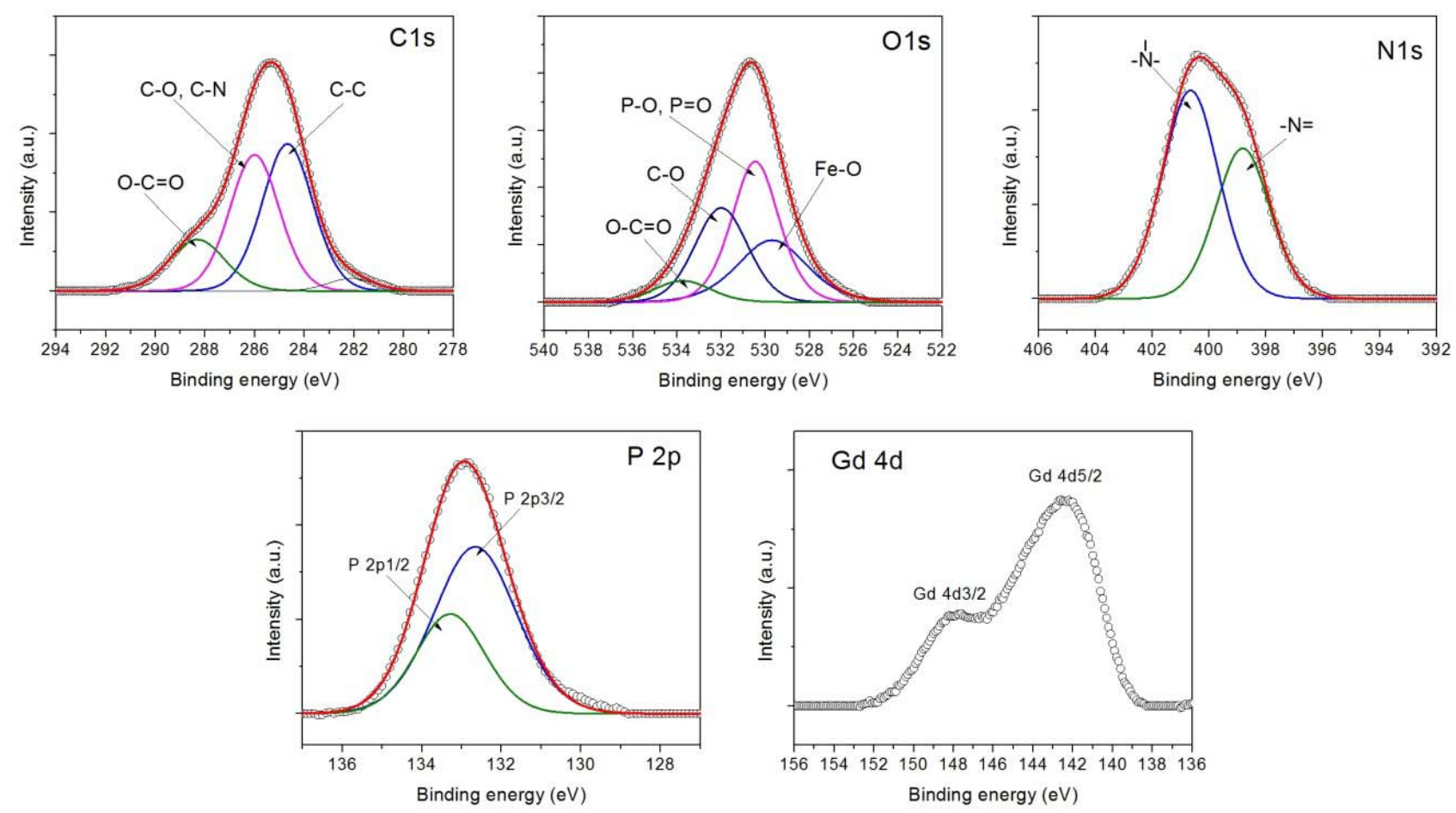

Fig. 4 - High resolution XPS spectra for C1s, O1s, N1s, P2p and Gd4d of the coating layer on iron oxide. 


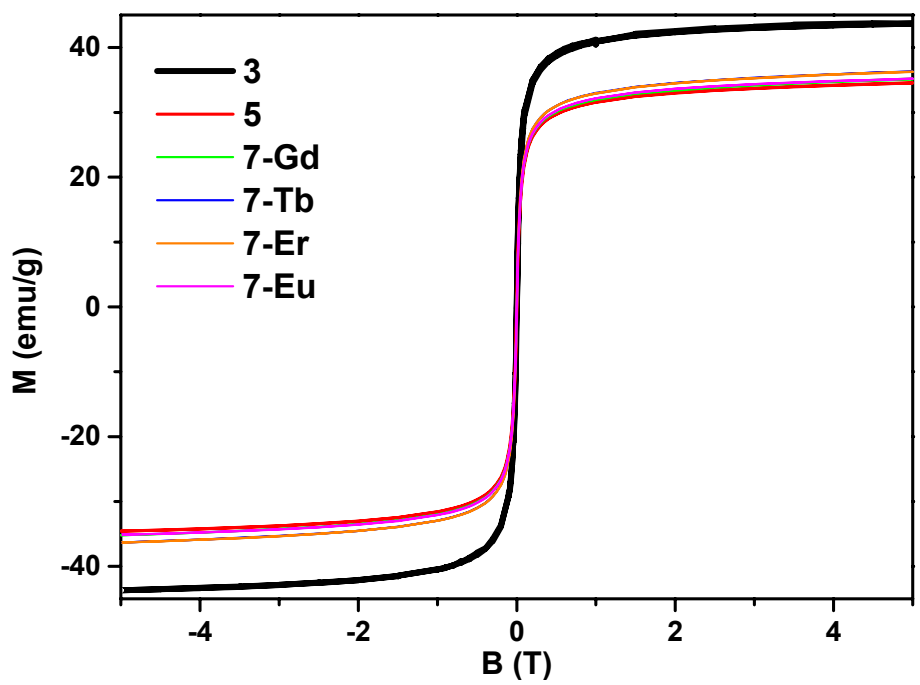

Fig. 5 - Magnetization versus applied magnetic field at room temperature of MNPs 3, 5, 7-Gd, 7-Tb, 7-Er and 7-Eu.

XPS is a powerful surface sensitive method for the investigation of surface chemical composition. In the Fig. 3 is shown the XPS survey spectrum of the sample which evidence the elements in the sample: Fe, C, N, P, O, Gd.

The high resolution XPS spectra for the elements from the coating layer of the magnetic iron oxide are presented in the Fig. 4. The spectra were deconvoluted into the components corresponding to particular bond types. The XPS spectra evidence the complexation of $\mathrm{Gd}^{3+}$ on the polymer coating layer.

The magnetic properties of all MNPs were investigated by magnetometry confirming superparamagnetic behaviour in each case. The values of saturation magnetization step down with the increasing the weight of the organic shell by polymerisation (Fig. 5), $44 \mathrm{emu} / \mathrm{g}$ (3) to $34 \mathrm{emu} / \mathrm{g}$ (5). The complexation reaction of the REs ions by the MNP 5 does not bring a drop in the saturation magnetization values of the resulting MNPs 7-Gd (35.1 emu/g), 7-Tb (36.1 emu/g), 7-Er (36.1 $\mathrm{emu} / \mathrm{g}$ ) and 7-Eu (35.4 emu/g). Regardless of all these differences, all MNPs exhibit saturation magnetizations high enough for the envisaged practical application in biomedicine.

\section{EXPERIMENTAL}

\section{Reagents}

All chemicals used were purchased from Sigma Aldrich and used as such.

\section{Synthesis of MNPs 3}

MNPs 1 were synthesized by the well-known coprecipitation method from $\mathrm{FeCl}_{3} * 6 \mathrm{H}_{2} \mathrm{O}$ and $\mathrm{FeCl}_{2} * 4 \mathrm{H}_{2} \mathrm{O}$ in the presence of aqueous ammonia with a ratio $2: 1$. The resulting magnetite particles were surface-functionalized in situ under argon by the addition of bis[2(methacryloyloxy)ethyl]phosphate $\mathbf{2}$ and strong stirring at $80{ }^{\circ} \mathrm{C}$ for $2 \mathrm{~h}$ (Scheme 1). After magnetic separation the particles were repeatedly washed with water and dried providing MNPs 3. They were used as such in free radical polymerization with VIm 4.

\section{Synthesis of MNPs 5 by polymerization of 1-} vinylimidazole in the presence of stabilized MNPs 3

MNPs 3 (2.32 g) were used for surface mediated free radical polymerization of VIm $4(7.1 \mathrm{~g}, 50 \mathrm{mmol})$ in the presence of AIBN $(0.19 \mathrm{~g}, 1.2 \mathrm{mmol})$ as radical initiator at $70{ }^{\circ} \mathrm{C}$ in dry dimethylformamide (DMF) for $24 \mathrm{~h}$ (Scheme 1). The MNPs-PVIm 5 were separated from the aqueous phase by magnetic separation with the help of a Nd magnet. They were washed successively with water, ethanol and acetone.

\section{Complexation of rare earth metals with MNP-PVIm 5}

The chelating reaction of REs ions $\left(\mathrm{Gd}^{3+}, \mathrm{Tb}^{3+}, \mathrm{Er}^{3+}\right.$ and $\left.\mathrm{Eu}^{3+}\right)$ at the MNP 5 surface took place in water at room temperature. MNP-PVIm $5(0.4 \mathrm{~g})$ were suspended in a solution of REs $\left(\mathrm{GdCl}_{3} \mathbf{6 - G d}(0.372 \mathrm{~g}, 1 \mathrm{mmol}) ; \mathrm{TbCl}_{3} \mathbf{6}\right.$ - $\mathbf{T b}$ (0.373 g, $1 \mathrm{mmol}) ; \mathrm{ErCl}_{3}$ 6-Er $(0.382 \mathrm{~g}, 1 \mathrm{mmol})$ and $\mathrm{EuCl}_{3}$ 6Eu $(0.366 \mathrm{~g}, 1 \mathrm{mmol}))$ in deionized water $(20 \mathrm{ml})$. The suspension was stirred at room temperature for 24 hours. The MNP doped with REs ions MNP 7-Gd, 7-Tb, 7-Er and 7-Eu were separated by an external magnet and washed several times with water to remove unreacted REs metals salt. The synthesized MNP were dried in the oven at $50{ }^{\circ} \mathrm{C}$ and then structurally characterized.

\section{Instrumentation}

Infrared absorption spectra in the $400-4000 \mathrm{~cm}^{-1}$ spectral range were recorded with a spectrophotometer JASCO FTIR6100 , using pressed pellets prepared from the MNPs powder embedded in $\mathrm{KBr}$. The morphology of the MNPs was determined by TEM using 1010 JEOL and Hitachi H9000NAR transmission electron microscopes. The chemical surface analysis for the functionalized magnetic nanoparticles was performed by X-ray Photoelectron Spectroscopy (XPS). 
XPS spectra were recorded using a XPS spectrometer SPECS equipped with a dual-anode X-ray source $\mathrm{Al} / \mathrm{Mg}$, a PHOIBOS 150 2D CCD hemispherical energy analyser and a multichanneltron detector with vacuum maintained at $1 \times 10^{-9}$ torr. The $\mathrm{AlK}_{\mathrm{K} \alpha} \mathrm{X}$-ray source $(1486.6 \mathrm{eV})$ operated at $200 \mathrm{~W}$ was used for XPS investigations. The XPS survey spectra were recorded at $30 \mathrm{eV}$ pass energy, $0.5 \mathrm{eV} / \mathrm{step}$. The highresolution spectra for individual elements were recorded by accumulating $10-15$ scans at $30 \mathrm{eV}$ pass energy and $0.1 \mathrm{eV} / \mathrm{step}$. The powdered sample was pressed on an indium foil to allow the XPS measurements. A cleaning of the samples surface was performed by argon ion bombardment $(500 \mathrm{~V})$. Data analysis and curve fitting was performed using Casa XPS software with a Gaussian-Lorentzian product function and a non-linear Shirley background subtraction. Magnetic measurements were performed at room temperature by using a Vibrating Sample Magnetometer Cryogenics.

\section{CONCLUSIONS}

In summary, new magnetic core shell nanoparticles $\mathbf{5}$ were synthesized consisting of a magnetite core and a poly(1-vinylimidazole) shell. MNP 1 stabilized by bis(methacryloyloxyethoxy) phosphate $\mathbf{2}$ as reactive alkene-containing anchor capable of joining the ongoing polymerization of 1 -vinylimidazole 4 in a type of copolymerization were used in this methodology. The poly(1vinylimidazole) coated MNPs 5 represent versatile magnetic nano-platforms capable of complexation REs metals ions (gadolinium 7-Gd, terbium 7-Tb, erbium 7-Er and europium 7-Eu). The results are attractive for application in biomedicine.

Acknowledgements. The authors wish to acknowledge, Dr. Cristian Leostean for conducting magnetic measurements and $\mathrm{PhD}$ student Sebastian Porav for TEM-EDX investigations. This work was supported by a grant of the Roumanian Ministry of Research and Innovation, project number PN-III-P1-1.2-PCCDI-2017-0769, contract no. 64, within PNCDI III.

\section{REFERENCES}

1. K. V. S. Ranganath and F. Glorius, Catal. Sci. Technol., 2011, 1, 13-22.

2. O. Veiseh, J. W. Gunn and M. Q. Zhang, Adv. Drug Deliver. Rev., 2010, 62, 284-304.

3. G. Liu, J. H. Gao, H. Ai and X. Y. Chen, Small, 2013, 9, 1533-1545.

4. L. H. Reddy, J. L. Arias, J. Nicolas and P. Couvreur, Chem. Rev., 2012, 112, 5818-5878.

5. S. Durr, C. Janko, S. Lyer, P. Tripal, M. Schwarz, J. Zaloga, R. Tietze and C. Alexiou, Nanotechnol. Rev., 2013, 2, 395-409.

6. S. Singamaneni, V. N. Bliznyuk, C. Binek and E. Y. Tsymbal, J. Mater. Chem., 2011, 21, 16819-16845.

7. L. Babes, B. Denizot, G. Tanguy, J. Jacques, L. Jenue and P. Jallet, J. Colloid Interface Sci., 1999, 212, 474-482.

8. M. Takafuji, S. Ide, H. Ihara and Z. Xu, Chem. Mater., 2004, 16,1977-1983.

9. A. Nan, R. Turcu, I. Craciunescu, O. Pana, H. Scharf and J. Liebscher, J. Polym. Sci. Polym. Chem., 2009, 47, 5379-5404.

10. S. Karsten, A. Nan, R. Turcu and J. Liebscher, J. Polym. Sci. Polym. Chem., 2012, 50, 3986-3995.

11. A. Nan, J. Leistner and R. Turcu, J. Nanopart. Res., 2013, 15, 1869.

12. C. Shan, Z. Ma, M. Tong and J. Ni, Water Res., 2015, 69, 252-260.

13. B. Zhao, M. He, B. Chen, H. Xu and B. Hu, Spectrochim. Acta B, 2018, 143, 32-41.

14. A. Pourjavadi, N. Safaie and S. H. Hosseini and C. Bennett, New J. Chem., 2016, 40, 1729-1736.

15. R. Zou, J. Xu, S. Kuffner, J. Becker, T. Li, X. Guan, X. Zhang, L. Li, M. A. Cohen Stuart and X. Guo, Energy Fuels, 2019, 33, 998-1006.

16. A. Nan, T. Radu and R. Turcu, RSC Advances, 2016, 6, 43330-43338.

17. R. Ramasamy, Armenian J. Phys., 2015, 8, 51-55.

18. A. Chowdhury and S. T. Thynell, Thermochimica Acta, 2006, 443, 159-172. 